\title{
Novel Raman Parametric Hybrid L-Band Amplifier with Four-Wave Mixing Suppressed Pump for Terabits Dense Wavelength Division Multiplexed Systems
}

\author{
Gaganpreet Kaur, ${ }^{1}$ Sanjay Sharma, ${ }^{2}$ and Gurmeet Kaur ${ }^{1}$ \\ ${ }^{1}$ Department of Electronics \& Communication Engineering, Punjabi University, Patiala, India \\ ${ }^{2}$ Department of Electronics \& Communication Engineering, Thapar University, Patiala, India \\ Correspondence should be addressed to Gaganpreet Kaur; preetkgagan@gmail.com
}

Received 29 September 2015; Revised 18 January 2016; Accepted 19 January 2016

Academic Editor: Mikhail Noginov

Copyright ( 92016 Gaganpreet Kaur et al. This is an open access article distributed under the Creative Commons Attribution License, which permits unrestricted use, distribution, and reproduction in any medium, provided the original work is properly cited.

\begin{abstract}
We demonstrate improved performance of parametric amplifier cascaded with Raman amplifier for gain of $54.79 \mathrm{~dB}$. We report amplification of L-band using $100 \times 10 \mathrm{Gbps}$ Dense Wavelength Division Multiplexed (DWDM) system with $25 \mathrm{GHz}$ channel spacing. The gain achieved is the highest reported so far with gain flatness of $3.38 \mathrm{~dB}$ without using any gain flattening technique. Hybrid modulated parametric pump is used for suppressing four-wave mixing (FWM) around pump region, resulting in improvement of gain flatness by $2.42 \mathrm{~dB}$. The peak to peak variation of gain is achieved less than $1.6 \mathrm{~dB}$. DWDM system with $16-$ channel, $25 \mathrm{GHz}$ spaced system has been analyzed thoroughly with hybrid modulated parametric pump amplified Raman-FOPA amplifier for gain flatness and improved performance in terms of BER and Q-factor.
\end{abstract}

\section{Introduction}

Fiber optical parametric amplifiers (FOPAs) are promising amplifiers for Wavelength Division Multiplexed (WDM) systems $[1,2]$ due to their capabilities to provide high gain and low-noise, broadband amplification $[1,3]$. The first demonstration of optical parametric amplification was reported by Stolen in low-loss optical fibers [4]. With advent of highly nonlinear fibers (HNLFs) [5], FOPAs gained considerable attention in long distance communication systems. Gain as high as $49 \mathrm{~dB}$ is demonstrated [6] using FOPAs whilst wavelength tuning flexibility of FOPAs helped in achieving gain over bandwidth above $200 \mathrm{~nm}$ [7]. Marhic et al. demonstrated on-off gain of $65 \mathrm{~dB}$ over bandwidth of more than $400 \mathrm{~nm}$ [8] using high power parametric pumps. Use of Photonic Crystal Fibers (PCFs), with nonlinearity coefficient $(\gamma)>$ 100 , added to wide gain capabilities of FOPAs. Parametric amplification using PCFs has been demonstrated to obtain continuous-wave bandwidth of more than $20 \mathrm{THz}$ [9]. Peak gain of $58 \mathrm{~dB}$ over wide gain bandwidth from 999 to $1139 \mathrm{~nm}$ [10] has been achieved using PCF based FOPAs. Feasibility of long haul, broadband communication using FOPAs has been demonstrated in multichannel DWDM systems too [11, 12]. An average gain of $22 \mathrm{~dB}$ using dual pump FOPA [11] has been reported by Chavez Boggio et al. for $10 \times 64 \mathrm{Gbps}$ system. Jazayerifar et al. [12] analytically proved the feasibility of long haul DWDM transmission up to $750 \mathrm{~km}$ systems using single pump FOPA.

Another class of fiber amplifiers capable of providing polarization-independent gain across a wide bandwidth is Raman amplifiers (RA). The gain of Raman amplifiers can be tailored to meet bandwidth requirements by choosing optimum number of pump lasers and their wavelengths appropriately. This flexibility makes them useful for entire communication bandwidth. Though Raman amplifiers provide distributed gain but noise due to pump-pump interactions, double Rayleigh backscattering [13] and amplified spontaneous noise (ASE) degrade the signal quality. Additionally, Double Raman Scattering (DRS) and low Raman gain coefficient limit the gain at every stage [14] of Raman amplification. 
Recently, Raman-FOPA (RA-FOPA) hybrid amplifiers have attracted considerable attention. The need for wideband, flat gain amplifier in WDM systems has motivated research interests towards RA-FOPA hybrid. The conventional RAFOPA hybrid amplifiers use Raman assisted parametric amplification wherein forward travelling parametric pump within an HNLF is augmented by a backward travelling Raman pump, usually in the same fiber [15]. This results in gain higher than sum of the individual gain contributions in certain regions of the spectrum $[15,16]$. In RA-FOPAs, long HNLF length is required to maximize the Raman gain. But at the same time, long HNLF lengths result in increased four-wave mixing cross talk products [17]. Though gain enhancement is achieved, in conventional RA-FOPAs, most energy from the Raman amplifier is trapped in the parametric pump at the output end of the amplifier. Wang and Wai [18] proposed a hybrid fiber Raman/parametric amplifier (HFRPA) constructed by cascading a FOPA after the RAFOPA. Peak small signal gain of $70 \mathrm{~dB}$ is achieved using $1 \mathrm{~km}$ HNLF with $1.5 \mathrm{~W}$ and $0.2 \mathrm{~W}$ Raman and parametric pump powers, respectively, for 8-channel WDM system. A net gain enhancement of $34 \mathrm{~dB}$ has been achieved over conventional RA-FOPA for the same parametric pump powers using the proposed HFRPA. Ummy et al. [19] demonstrated an extended flat gain of $15 \mathrm{~dB}$ with gain ripple of $5 \mathrm{~dB}$, using combined Raman and parametric interaction in HNLF. Peiris et al. [20] demonstrated hybrid Raman-Optical Parametric Amplifier (HROPA) in tandem configuration for extended bandwidth, with gain more than $20 \mathrm{~dB}$ and extended gain bandwidth of $170 \mathrm{~nm}$. The gain ripple achieved is less than $4 \mathrm{~dB}$. In tandem configuration, transmitted frequencies are divided into two subbands and a common, single pump FOPA is used for amplification of each. Cross talk generated due to idlers is controlled through a multiplexer filter transfer function. In [21], improved performance for WDM system comprising of ten, $100 \mathrm{GHz}$ spaced DFB lasers has been achieved using RA-FOPA with net gain of $20 \mathrm{~dB}$ and gain ripple of $1.9 \mathrm{~dB}$. Different optical amplifiers and their hybrids have been investigated over the time, to exploit the available optical communication bands, namely, S, C, and L. Though C/L-bands are dominated by EDFAs and their hybrids [22, 23], Raman amplifiers [24] as well as FOPAs [3, 25] can be tuned across any band. Kaur et al. [26] demonstrated flat gain of $24.3 \mathrm{~dB}$ over bandwidth of $220 \mathrm{~nm}$ using RA and multisection FOPA cascade. Akasaka et al. [27] demonstrated S-band amplification and wavelength conversion using RAFOPA. Freitas et al. [28] demonstrated $10 \mathrm{~dB}$ gain enhancement in S-band and S/C-band wavelength conversion by simultaneous Raman as well as parametric amplification. We have previously demonstrated gain of more than $14 \mathrm{~dB}$ in Lband with ripple of less than $1.4 \mathrm{~dB}$ using RA-FOPA cascade with a gain flattening filter [29].

In this paper, we present successful analysis of a Raman-FOPA cascade hybrid amplifier for achieving flat gain in L-band, DWDM system without any gain flattening/compensating technique. The RA-FOPA cascade has been chosen over RA assisted FOPA to fully exploit the flexibilities to tune Raman and FOPA pumps such that gain is provided in separate regions of bandwidth. To achieve

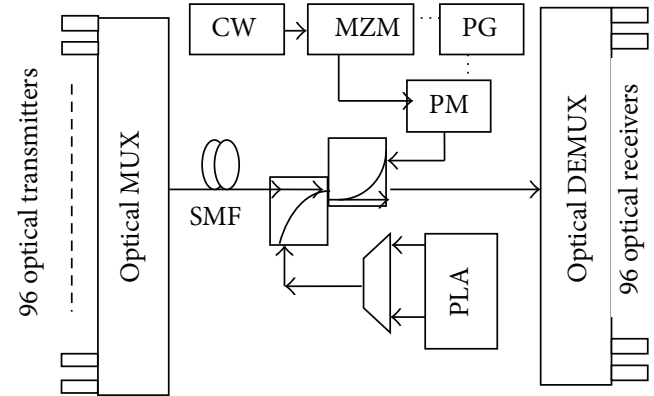

Figure 1: Experimental setup for Raman-FOPA with hybrid modulator for FWM suppression. PLA: pump laser array, PM: phase modulator, MZM: Mach Zender modulator, CW: continuous-wave laser, and PG: pulse generator.

flat gain, we propose using FWM suppression at parametric pumps instead of applying external modulators at each transmitter. Use of MZM-PM hybrid modulator for parametric pump to achieve FWM suppression has been proposed.

\section{Experimental Setup}

Experimental setup consists of 100 DWDM channels spaced $25 \mathrm{GHz}$ apart covering L-band frequencies from $187 \mathrm{THz}$ to 189.475 THz as shown in Figure 1.

The proposed system uses Raman-FOPA cascade as inline amplifier after traversing $50 \mathrm{~km}$ Single Mode Fiber (SMF). The dispersion of SMF is compensated by $10 \mathrm{~km}$ fiber used for Raman amplification with its dispersion $(D)=$ $-90 \mathrm{ps} / \mathrm{km} / \mathrm{nm}$. Raman amplified signal is then launched into short length HNLF of $0.2 \mathrm{~km}$ for parametric amplification. The CW parametric pump used is hybrid modulated using MZM-PM modulated RZ signal.

Single HNLF fiber section of length " $L$ " results in gain $G$ given by

$$
G=1+\left(\frac{\gamma P}{g} \sinh (g L)\right)^{2}
$$

where $g=\sqrt{(\gamma P)^{2}-(K / 2)^{2}}$ for single pump FOPA, where $K=\Delta \beta+2 \gamma P$ and

$$
\Delta \beta=2 \sum_{m=1}^{\infty} \frac{\beta_{2 \mathrm{~m}}}{(2 \mathrm{~m}) !}\left[\Omega^{2 \mathrm{~m}}-\left(\omega_{p}\right)^{2 \mathrm{~m}}\right], \quad \Omega=\omega_{s}-\omega_{p}
$$

From theory of Raman scattering in optical fibers, Raman interactions are expressed by [30]

$$
\delta=-\left(\frac{3 \omega_{1}}{8 n c A_{\mathrm{eff}}}\right) \chi_{3}^{\prime \prime}(\Omega),
$$

where $n$ is the refractive index of the core, $\Omega$ is the frequency deviation from pump frequency, and $\chi_{3}^{\prime \prime}(\Omega)$ is the imaginary 
part of the third-order susceptibility resulting in set of equations expressed as

$$
\begin{aligned}
& \frac{\partial A_{3}}{\partial z}=2 q A_{3}+q A_{4}^{*} e^{-i K z}, \\
& \frac{\partial A_{4}^{*}}{\partial z}=-2 q A_{4}^{*}-q A_{3} e^{i K z}
\end{aligned}
$$

for signal and idler, respectively.

Here, $q=(i \gamma+\delta) P_{0}$ affects the four-wave mixing interaction of signal waves causing significant gain variation due to phase modulation originating from pump interactions [31]. Gao et al. [32] analyzed the phase mismatch conditions for parametric gain and derived the dependence of parametric gain on the real part of the complex Raman susceptibility. Use of tunable NRZ signals is suggested for reduced susceptibility of gain saturation in Raman-FOPA hybrid [33]. The real part of Raman susceptibility strongly affects peak parametric gain in case of single pump FOPA [34] in dispersion shifted fibers. In case of broadband amplification, Raman gain can cause significant spectral distortions [35] in RA-FOPA hybrid. In our analysis, we assume gain variations due to Raman susceptibility relatively small in comparison to nonlinear parametric gain as HNLF with high " $\gamma$."

To optimize use of pumps in RA-FOPA cascade, it has been considered that, for bandwidths of order of $200 \mathrm{~nm}$, the FOPA gain significantly overlaps with the pump-induced Raman gain which peaks at about $110 \mathrm{~nm}$ from the pump on the longer wavelength region of spectrum [36]. To keep this to a minimum, we choose Raman pumps from $1485 \mathrm{~nm}$ to $1513 \mathrm{~nm}$ with varying pump powers $(200-350 \mathrm{~mW})$ such that gain is more at lower frequency end from $187 \mathrm{THz}$ to $188.725 \mathrm{THz}$. Figure 2 shows the power and wavelength of Raman pumps. Pump wavelengths have been carefully optimized to achieve flat gain. For band of frequencies higher than $188.725 \mathrm{THz}$, parametric amplification is used with pump tuned at $1583.56 \mathrm{~nm}$. So the pump difference of at least $70 \mathrm{~nm}$ is maintained between Raman pumps and parametric pump to keep pump-pump FWM cross talk to a minimum. Moreover, peak gain regions of both Raman and FOPA fall at different band of frequencies in the output spectrum. Raman pump is tuned to provide gain in lower frequency region while parametric amplification provides the maximum gain at higher end of frequencies.

The proposed schematic is governed by

$$
G_{\text {total }}=G_{\mathrm{Raman}} * G_{\mathrm{FOPA}} \text {, }
$$

where $G_{\text {Raman }}$ and $G_{\mathrm{FOPA}}$ are the gain of parametric sections. Raman gain in $\mathrm{dB}$ is $10 \cdot \log _{10}\left(\exp \left(g_{R} \cdot P \cdot L_{\text {eff }} / A_{\text {eff }}-\alpha L\right)\right)$.

The parameters used for Raman fiber serving as DCF and HNLF used for parametric amplifier are listed in Table 1.

\section{Results and Discussions}

In this work, we propose achieving flat gain for Terabits capacity system implemented as $100 \times 10$ channels with $25 \mathrm{GHz}$ spaced RZ signals. RA-FOPA cascade is used as inline amplifier and dispersion compensation has been combined
TABLE 1: Values of parameters used for HNLF parametric amplifier and DCF in Raman amplifier.

\begin{tabular}{lc}
\hline Parameter & Value \\
\hline DCF length & $10 \mathrm{~km}$ \\
Dispersion (DCF) & $-90 \mathrm{ps} / \mathrm{km} / \mathrm{nm}$ \\
DCF attenuation & $0.5 \mathrm{~dB} / \mathrm{km}$ \\
Effective area & $30 \mu \mathrm{m}^{2}$ \\
HNLF length & $0.2 \mathrm{~km}$ \\
HNLF effective area & $11 \mu \mathrm{m}^{2}$ \\
Dispersion (HNLF ) & $2 \mathrm{ps} / \mathrm{km} / \mathrm{nm}$ \\
Nonlinear coefficient $(\gamma)$ & 11.45 \\
HNLF attenuation & $0.8 \mathrm{~dB} / \mathrm{km}$ \\
\hline
\end{tabular}

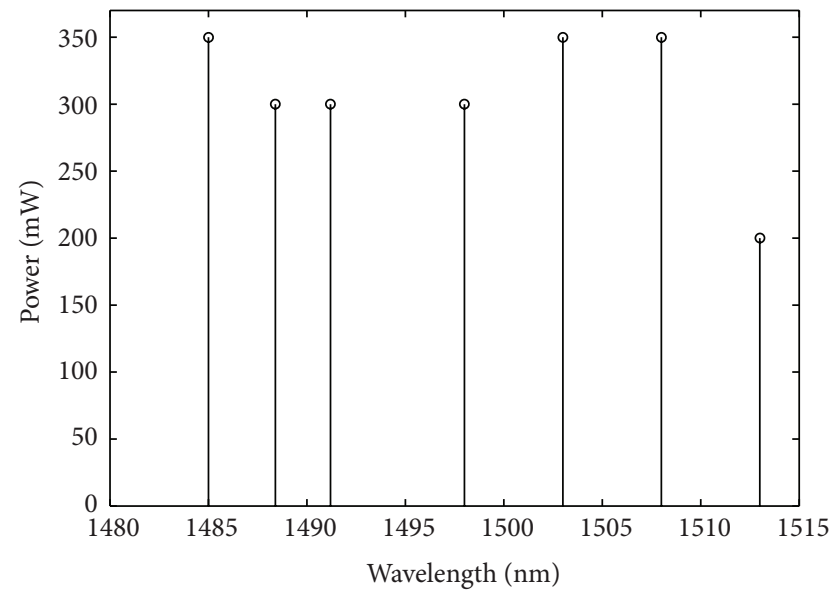

FIGURE 2: Spectral allocation of Raman pumps.

in Raman amplifying fiber medium itself. Use of single pump parametric amplifiers for broadband amplification has been reported previously $[12,19,26]$. But with increased number of channels in DWDM systems, high pump powers of the order of $200 \mathrm{~mW}$ are required to overcome the cross talk due to parametric amplification [12]. High pump power coupled with narrow channel spacing poses serious challenge to achievement of flat gain over the entire band. Due to relatively high pump power, the frequencies in immediate vicinity of pump frequency tend to have much higher gain than frequencies at the extremes of the band. This gives high ripple in the amplifier gain. To make the gain flat, we propose modulating the parametric pump using MZM-PM hybrid modulation. The resultant modulated pump pulse is pulse spread across band of frequencies with its center at the pump frequency.

Use of hybrid modulated parametric pump to suppress FWM results in flat gain when compared to similar system employing unmodulated CW parametric pump. Results of the implemented system using MZM-PM hybrid modulated pump indicate a nearly flat gain as shown in Figure 3. The fiber serving as medium for Raman amplification has been used for dispersion compensation [14]. Results show a peak gain of $57.24 \mathrm{~dB}$ for CW parametric pump, whereas using MZM-PM hybrid modulated parametric pump peak gain of $54.79 \mathrm{~dB}$ is 


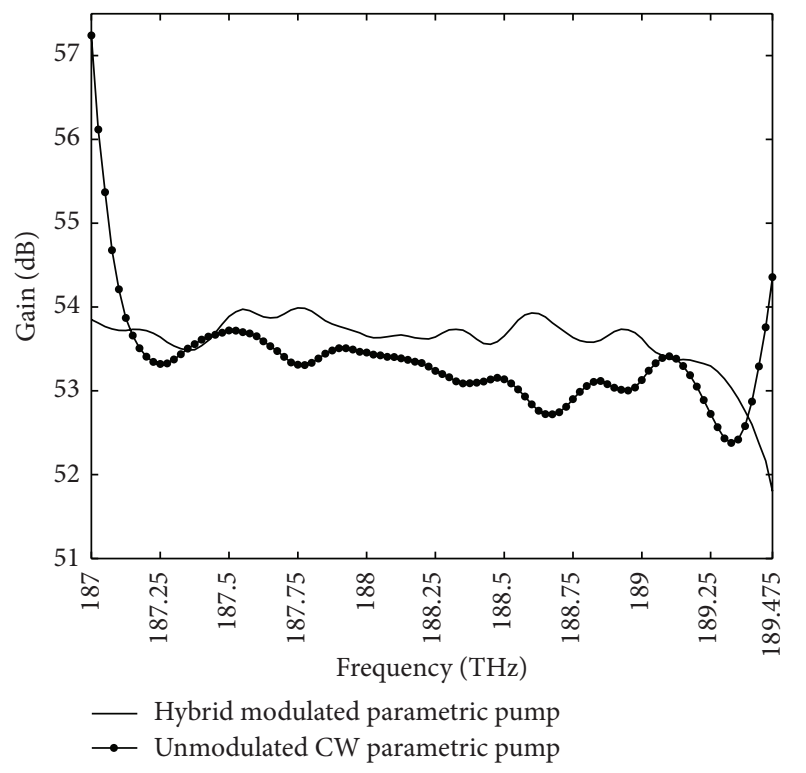

FIGURE 3: Gain comparison for 100-channel RA-FOPA at $10 \mathrm{Gbps}$ with hybrid modulated parametric pump (solid curve) versus unmodulated CW parametric pump.

achieved. Though peak gain reduces using hybrid modulated pump but gain flatness improves, when hybrid modulated parametric pump is used, gain ripple is reduced to $3.38 \mathrm{~dB}$ from $5.8 \mathrm{~dB}$ in case of unmodulated CW parametric pumped system. Also, higher average gain of $53.6 \mathrm{~dB}$ is achieved for hybrid modulated parametric pump against average gain of $53.27 \mathrm{~dB}$ with $\mathrm{CW}$ parametric pump (excluding the peak gain at extreme right shown by dotted curve in Figure 3).

Figure 4 shows gain comparison using proposed RAFOPA amplifier for L-band with unmodulated CW parametric pump versus hybrid modulated parametric pump at $40 \mathrm{Gbps}$. When hybrid modulation is applied to parametric pump, the maximum gain is $26.88 \mathrm{~dB}$ while minimum gain is $19.23 \mathrm{~dB}$ giving ripple of $7.65 \mathrm{~dB}$ while in the absence of any modulation parametric pump results in maximum gain of $28.12 \mathrm{~dB}$ while minimum gain is $18.22 \mathrm{~dB}$ resulting in ripple of $9.91 \mathrm{~dB}$. So we achieve flatness improvement of $2.26 \mathrm{~dB}$. With increase in data rate, net gain has decreased and the gain ripple has increased. But use of hybrid modulated parametric pump definitely gives better performance over conventional parametric pumped FOPA even at high data rates.

To analyze FWM suppression in proposed system, we simulate a 16-channel system at $25 \mathrm{GHz}$ channel spacing extending from $187.1 \mathrm{THz}$ to 187.475 . The parametric pump with $10 \mathrm{~mW}$ power is fixed at frequency of $187.323 \mathrm{THz}$ while Raman pump is located at $1501.6 \mathrm{~nm}$.

It can be well observed from gain results in Figure 5 that when we use hybrid modulated parametric pump the peak gain is decreased and low gain regions slightly raised which helps reduce the gain extremes. Due to use of relatively high power pump, the FWM effect results in sharp gain at channel frequencies close to pump at $187.32 \mathrm{THz}$ while gain diminishes towards the high and low extremes of the bandwidth. But when we modulate the parametric pump

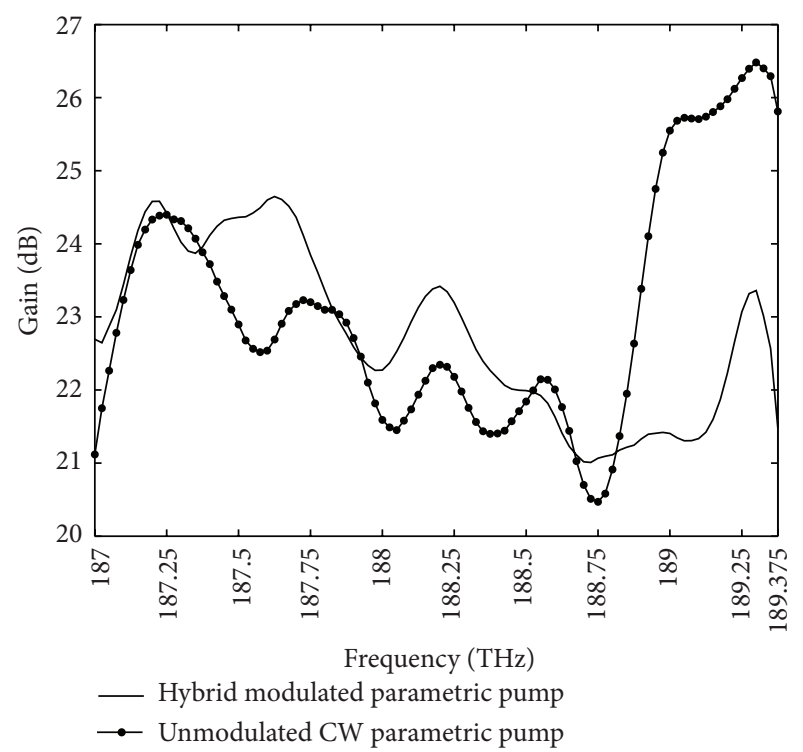

FIGURE 4: Gain comparison for RA-FOPA at 40 Gbps with hybrid modulated parametric pump (solid) and no modulation CW pump (dotted).

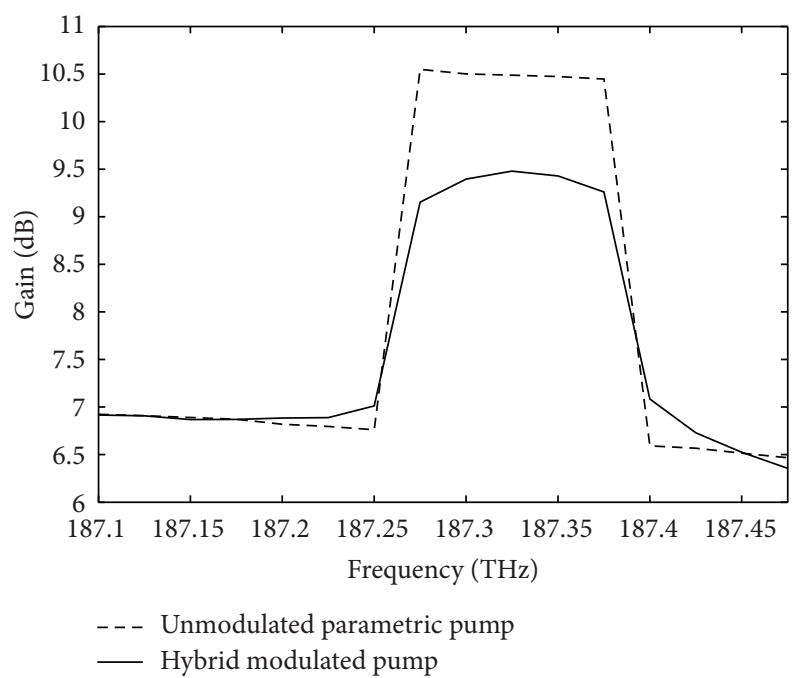

FIGURE 5: Gain of 16-channel system with and without hybrid modulator.

using MZM-PM, resulting pulse is not as sharp but is a pulse spread over range of frequencies around the pump frequency with power in side bands diminishing slowly. Additionally, due to the phase modulation, the peak power is further decreased as phase difference increases, resulting in lower FWM efficiency. This lowering of peak power in vicinity of parametric pump results in lesser gain ripple between highest and lowest gain achieved across the entire bandwidth.

The quality of received signal can be well observed from eye diagrams in Figures 6(a) and 6(b). Figure 6(a) shows eye opening when hybrid modulated parametric pump is used. The minimum eye opening is $1.3 \times 10^{-4}$ while as seen 


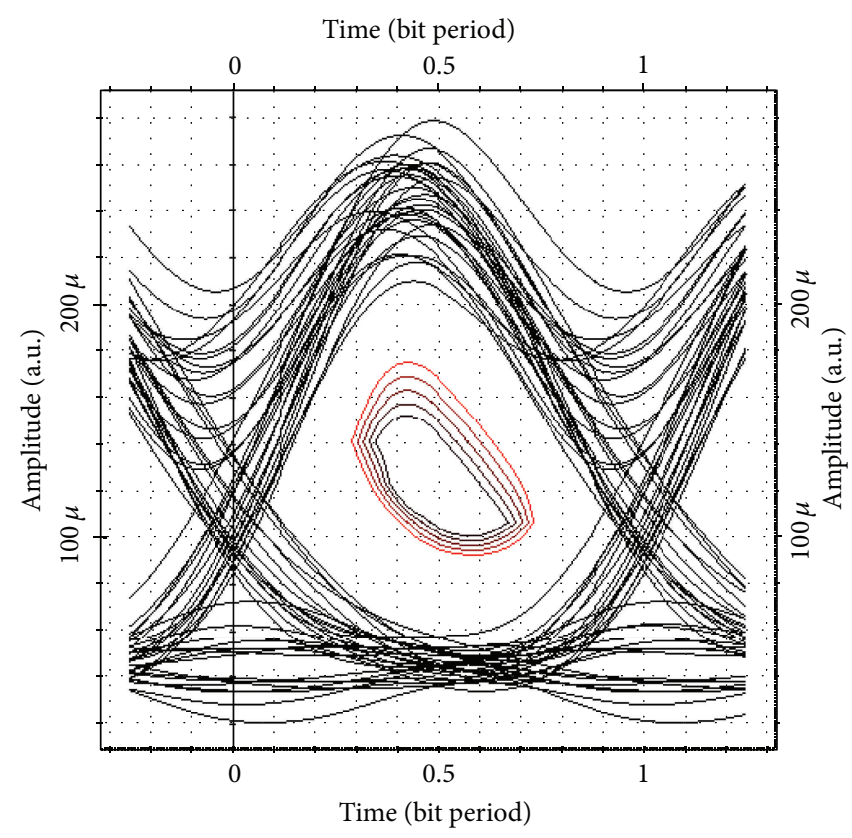

(a)

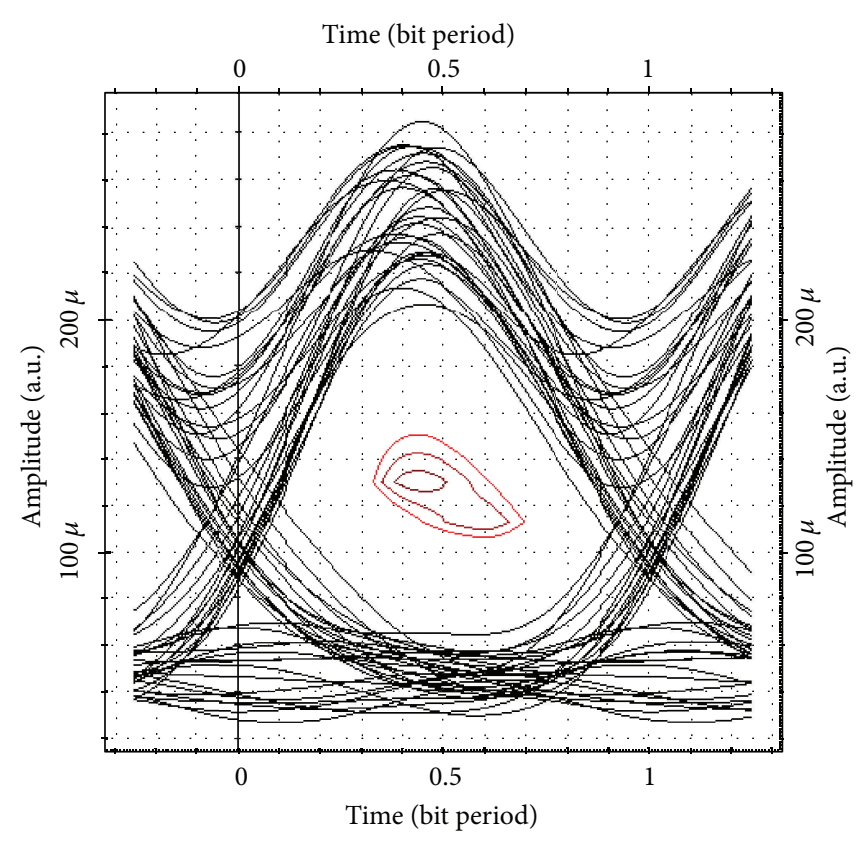

(b)

FIGURE 6: (a) BER pattern and eye diagram for hybrid modulated parametric pump based system. (b) BER pattern and eye diagram for unmodulated CW parametric pump.

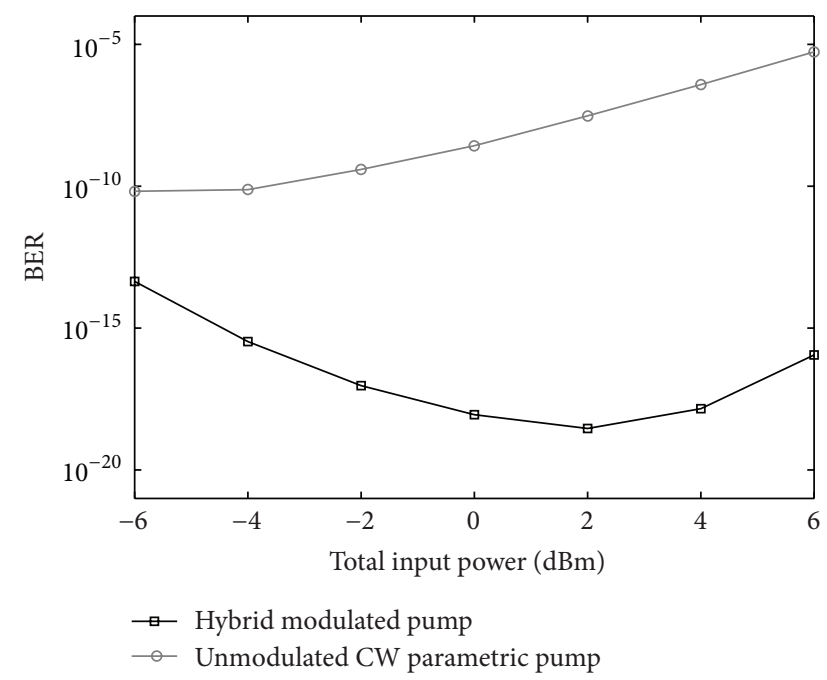

FIGURE 7: BER of hybrid modulated system versus unmodulated parametric pump.

in Figure 6(b) for system with CW parametric pump eye opening is $1.1 \times 10^{-4}$.

BER analysis in Figure 7 shows a significant improvement with use of hybrid modulator for parametric pump. Figure 7 shows improvement in BER to order of $10^{-19}$ from $10^{-12}$ at $-2 \mathrm{dBm}$ of transmitter input power, which is minimum BER achieved in both cases, respectively.

Results of the $Q$-factor variation in Figure 8 confirm improved performance using the proposed hybrid. Use of

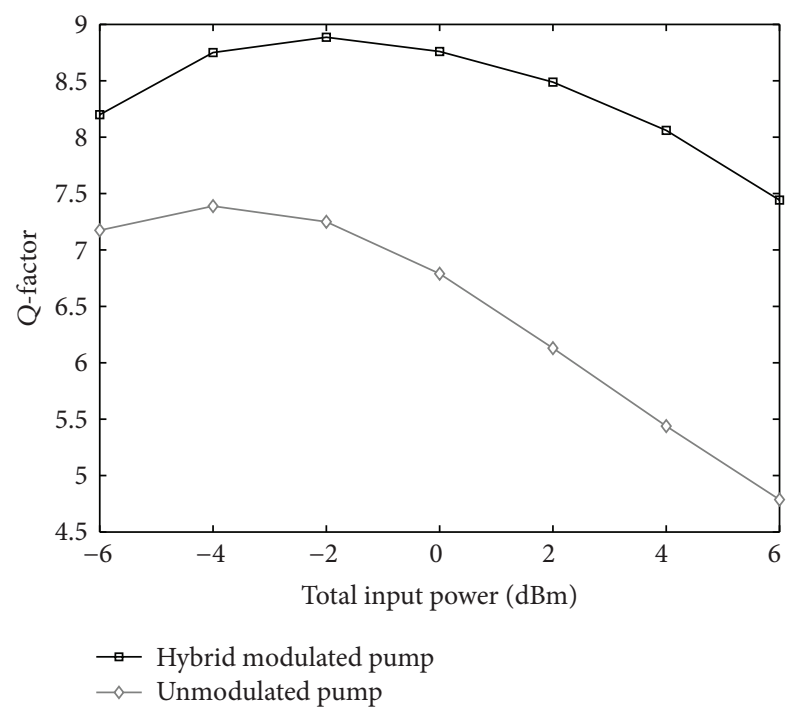

FIGURE 8: Q-factor of hybrid modulated system versus unmodulated parametric pump.

hybrid modulator improves quality factor from 7.2 to nearly 9 at total input power of $-2 \mathrm{dBm}$.

Another significant parameter in determining performance of an optical communication system is dispersion. For the proposed system, dispersion in the transmitted signals has been analyzed briefly. The dispersion analysis result in Figure 9 shows that increase in dispersion of the transmitted signals when employing hybrid modulated parametric pump 


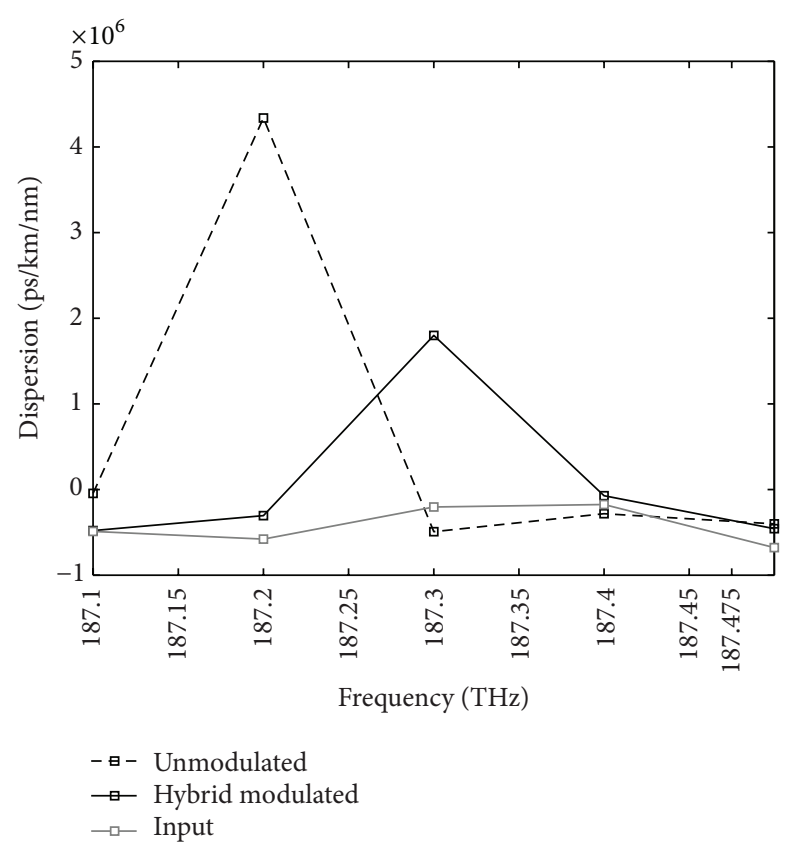

FIGURE 9: Dispersion variation of hybrid modulated parametric pump versus unmodulated parametric pump.

is much less than the system which uses unmodulated CW parametric pump.

The solid curve in Figure 9 shows lesser dispersion increase with use of proposed hybrid modulator.

So, results confirm improved performance of DWDM system with RA-FOPA cascade hybrid amplifier when using hybrid modulated parametric pump.

\section{Conclusion}

We have proposed a novel idea to modulated parametric pump using MZM-PM hybrid modulation to achieve flat gain for RA-FOPA cascaded amplifier. The gain achieved has been the highest reported so far using RA-FOPA hybrid used as inline amplifier for 100-channel L-band DWDM system. Never before for RA-FOPA hybrid dispersion compensation is reportedly combined with Raman amplifier fiber medium making it more economical and compact. Achieved gain of $54.79 \mathrm{~dB}$, with gain ripple of $3.38 \mathrm{~dB}$, is the best reported result. No gain flattening or compensating technique has been employed. Previous best gain in L-band for 100channel, $25 \mathrm{GHz}$ spaced DWDM system at $10 \mathrm{Gbps}$ has been reported as $>24 \mathrm{~dB}$ using Er-Yb codoped waveguide amplifier (EYDWA) and a semiconductor optical amplifier (SOA) hybrid [36] as booster amplifier.

Our work is motivated from hybrid modulation techniques used to suppress FWM cross talk. Recently, FWM suppression techniques for WDM systems have been proposed $[37,38]$. But as a general approach, hybrid modulators have only been applied to transmitters for FWM suppression [37]. This significantly adds to size and cost of system. So, we propose using hybrid modulator for $\mathrm{CW}$ parametric pump to suppress FWM generated idlers and hence achieving improved gain flatness in RA-FOPA hybrid. This has never been done previously. Results achieved are the best reported to date for L-band DWDM amplification.

Future scope of proposed RA-FOPA can be viewed multidimensionally. FWM has been the most detrimental effect for narrow spaced DWDM systems [34]. We have exploited FWM for amplification in DWDM systems in combination with partial suppression to facilitate flat gain. Our proposed system can be explored to achieve significant results with polarization based FWM suppression techniques for further gain enhancement. Additionally, novel techniques for parametric pump modulation in other existing hybrid parametric amplifier configurations [39] may help improvise the gain in WDM systems.

Another characteristic of FOPA is dependence of gain on fiber properties. Advent of novel fiber [40] design with reduced sensitivity of fiber dispersion to diameter fluctuations would help achieve more dependable results for broadband amplification using parametric amplifiers.

In conclusion, thorough investigation has been done in direction to establish use of RA-FOPA hybrid amplifiers across all bands of communication [3, 26-29], namely, S, C, and $\mathrm{L}$ with good gain and wide gain bandwidth. Exploring the possibilities of using proposed RA-FOPA in combination with existing/novel techniques for gain enhancement may help identify a true broadband amplifier capable of providing flat gain across entire communication band for evolving WDM systems.

\section{Conflict of Interests}

The authors declare that there is no conflict of interests regarding the publication of this paper.

\section{References}

[1] M. E. Marhic, Fiber Optical Parametric Amplifiers, Oscillators and Related Devices, Cambridge University Press, Cambridge, UK, 1st edition, 2008.

[2] B. P.-P. Kuo, P. C. Chui, and K. K.-Y. Wong, "A comprehensive study on crosstalk suppression techniques in fiber optical parametric amplifier by modulation format," IEEE Journal on Selected Topics in Quantum Electronics, vol. 14, no. 3, pp. 659665, 2008.

[3] J. Hansryd, P. A. Andrekson, M. Westlund, J. Li, and P.-O. Hedekvist, "Fiber-based optical parametric amplifiers and their applications," IEEE Journal on Selected Topics in Quantum Electronics, vol. 8, no. 3, pp. 506-520, 2002.

[4] R. H. Stolen, "Phase-matched stimulated four-photon mixing in silica fiber waveguides," IEEE Journal of Quantum Electronics, vol. 11, no. 3, pp. 100-103, 1975.

[5] M. J. Holmes, D. L. Williams, and R. J. Manning, "Highly nonlinear optical fiber for all optical processing applications," IEEE Photonics Technology Letters, vol. 7, no. 9, pp. 1045-1047, 1995.

[6] M. E. Marhic, N. Kagi, T.-K. Chiang, and L. G. Kazovsky, "Broadband fiber optical parametric amplifiers," Optics Letters, vol. 21, no. 8, pp. 573-575, 1996.

[7] J. Hansryd and P. A. Andrekson, "Broad-band continuouswave-pumped fiber optical parametric amplifier with 49-dB 
gain and wavelength-conversion efficiency,' IEEE Photonics Technology Letters, vol. 13, no. 3, pp. 194-196, 2001.

[8] M. E. Marhic, K. K.-Y. Wong, and L. G. Kazovsky, "Wideband tuning of the gain spectra of one-pump fiber optical parametric amplifiers," IEEE Journal on Selected Topics in Quantum Electronics, vol. 10, no. 5, pp. 1133-1141, 2004.

[9] A. Mussot, A. Kudlinski, R. Habert et al., "20 THz-bandwidth continuous-wave fiber optical parametric amplifier operating at $1 \mu \mathrm{m}$ using a dispersion-stabilized photonic crystal fiber," Optics Express, vol. 20, no. 27, pp. 28906-28911, 2012.

[10] L. Zhang, S. Yang, X. Wang et al., "Photonic crystal fiber based wavelength-tunable optical parametric amplifier and picosecond pulse generation," IEEE Photonics Journal, vol. 6, no. $5,2014$.

[11] J. M. Chavez Boggio, P. Dainese, and H. L. Fragnito, "Performance of a two-pump fiber optical parametric amplifier in a $10 \mathrm{~Gb} / \mathrm{s} \times 64$ channel dense wavelength division multiplexing system," Optics Communications, vol. 218, no. 4-6, pp. 303-310, 2003.

[12] M. Jazayerifar, S. Warm, R. Elschner et al., "Performance evaluation of DWDM communication systems with fiber optical parametric amplifiers," Journal of Lightwave Technology, vol. 31, no. 9, pp. 1454-1461, 2013.

[13] P. B. Hansen, L. Eskildsen, A. J. Stentz et al., "Rayleigh scattering limitations in distributed Raman pre-amplifiers," IEEE Photonics Technology Letters, vol. 10, no. 1, pp. 159-161, 1998.

[14] M. N. Islam, "Raman amplifiers for telecommunications," IEEE Journal on Selected Topics in Quantum Electronics, vol. 8, no. 3, pp. 548-559, 2002.

[15] C. J. S. de Matos, D. A. Chestnut, P. C. Reeves-Hall, and J. R. Taylor, "Continuous-wave-pumped Raman assisted fiber optical parametric amplifier and wavelength converter in conventional dispersion-shifted fiber," Optics Letters, vol. 26, no. 20, pp. 15831585, 2001.

[16] S. H. Wang, L. Xu, P. K. A. Wai, and H. Y. Tam, "6.4-dB enhancement of the gain of a raman-assisted fiber optical parametric amplifier over the sum of the gains of individual amplifiers," in Proceedings of the OFC/NFOEC Conference on Optical Fiber communication/National Fiber Optic Engineers Conference, pp. 1-3, IEEE, San Diego, Calif, USA, February 2008.

[17] M. Jamshidifar, A. Vedadi, and M. E. Marhic, "Reduction of four-wave-mixing crosstalk in a short fiber-optical parametric amplifier," IEEE Photonics Technology Letters, vol. 21, no. 17, pp. 1244-1246, 2009.

[18] S. H. Wang and P. K. A. Wai, "Gain enhancement in hybrid fiber Raman/parametric amplifiers," in Proceedings of the Conference on Lasers and Electro-Optics (CLEO '10) and Quantum Electronics and Laser Science Conference (QELS '10), pp. 1-2, San Jose, Calif, USA, May 2010.

[19] M. A. Ummy, M. F. Arend, L. Leng, N. Madamopoulos, and R. Dorsinville, "Extending the gain bandwidth of combined raman-parametric fiber amplifiers using highly nonlinear fiber," Journal of Lightwave Technology, vol. 27, no. 5, pp. 583-589, 2009.

[20] S. Peiris, N. Madamopoulos, N. Antoniades, D. Richards, M. A. Ummy, and R. Dorsinville, "Engineering an extended gain bandwidth hybrid Raman-optical parametric amplifier for next generation CWDM PON," Journal of Lightwave Technology, vol. 32, no. 5, Article ID 6684559, pp. 939-946, 2014.

[21] M. F. C. Stephens, I. D. Phillips, P. Rosa, P. Harper, and N. J. Doran, "Improved WDM performance of a fibre optical parametric amplifier using Raman-assisted pumping," Optics Express, vol. 23, no. 2, pp. 902-911, 2015.

[22] S. Singh and R. S. Kaler, "Flat-gain L-band raman-EDFA hybrid optical amplifier for dense wavelength division multiplexed system," IEEE Photonics Technology Letters, vol. 25, no. 3, pp. 250-252, 2013.

[23] S. Singh and R. S. Kaler, "Flat-gain characteristics of C+L splitband hybrid waveguide amplifier for dense wavelength division multiplexed system at reduced channel spacing," Optoelectronics and Advanced Materials-Rapid Communications, vol. 8, no. 9-10, pp. 980-984, 2014.

[24] M. N. Islam, Ed., Raman Amplifiers for Telecomunications1: Physical Principles, Springer, New York, NY, USA, 2004.

[25] M. E. Marhic, K. K.-Y. Wong, G. Kalogerakis, and L. G. Kazovsky, "Toward practical fiber optical parametric amplifiers and oscillators," Optics \& Photonics News, vol. 20, 2004.

[26] G. Kaur, G. Kaur, and S. Sharma, "Multisection optical parametric-Raman hybrid amplifier for terabit+ WDM systems," Journal of Modern Optics, 2015.

[27] Y. Akasaka, K. K. Y. Wong, M.-C. Ho, M. E. Marhic, L. G. Kazovsky, and Y. Akasaka, "Novel S-band amplification and wavelength conversion technique using dual nonlinear phenomena," in Proceedings of the Optical Fiber Communication Conference and Exhibit (OFC '01), IEEE, Anaheim, Calif, USA, March 2001.

[28] J. F. L. Freitas, M. B. Costa e Silva, S. R. Lüthi, and A. S. L. Gomes, "Raman enhanced parametric amplifier based SC band wavelength converter: experiment and simulations," Optics Communications, vol. 255, no. 4-6, pp. 314-318, 2005.

[29] G. Kaur, G. Kaur, and S. Sharma, "Enhanced gain using Raman FOPA hybrid Amplifier for L band 96 X100 Gbps DWDM systems," in Proceedings of the IEEE Conference on Next Generation Computing Technologies, IEEE, September 2015.

[30] F. Yaman, Q. Lin, S. Radic, and G. E. Agrawal, "Impact of pumpphase modulation on dual-pump fiber-optic parametric amplifiers and wavelength converters," IEEE Photonics Technology Letters, vol. 17, no. 10, pp. 2053-2055, 2005.

[31] E. A. Golovchenko, P. V. Mamyshev, A. N. Pilipetskii, and E. M. Dianov, "Mutual influence of the parametric effects and stimulated Raman scattering in optical fibers," IEEE Journal of Quantum Electronics, vol. 26, no. 10, pp. 1815-1820, 1990.

[32] M. J. Gao, C. Jiang, and W. Hu, "Dual-pump broadband fiber optical parametric amplifier with a three-section photonic crystal fiber scheme," in Passive Components and Fiber-Based Devices, vol. 5623 of Proceedings of SPIE, pp. 300-308, Beijing, China, January 2005.

[33] A. S. Y. Hsieh, S. G. Murdoch, S. Coen, R. Leonhardt, and J. D. Harvey, "Influence of Raman susceptibility on optical parametric amplification in optical fibers," Optics Letters, vol. 32, no. 5, pp. 521-523, 2007.

[34] M. E. Marhic, P. A. Andrekson, P. Petropoulos, S. Radic, C. Peucheret, and M. Jazayerifar, "Fiber optical parametric amplifiers in optical communication systems," Laser and Photonics Reviews, vol. 9, no. 1, pp. 50-74, 2015.

[35] M.-C. Ho, K. Uesaka, M. Marhic, Y. Akasaka, and L. G. Kazovsky, "200-nm-bandwidth fiber optical amplifier combining parametric and Raman gain," IEEE Journal of Lightwave Technology, vol. 19, no. 7, pp. 977-982, 2001.

[36] S. Singh and R. S. Kaler, "Novel optical flat-gain hybrid amplifier for dense wavelength division multiplexed system," IEEE Photonics Technology Letters, vol. 26, no. 2, pp. 173-176, 2014. 
[37] H. Abd, N. M. Din, M. H. Al-Mansoori, F. Abdullah, and H. A. Fadhil, "Four-wave mixing crosstalk suppression based on the pairing combinations of differently linear-polarized optical signals," Scientific World Journal, vol. 2014, Article ID 243795, 10 pages, 2014.

[38] G. Kaur and M. S. Patterh, "Suppression of four wave mixing in wavelength division multiplexed system with hybrid modules," Optik, vol. 125, no. 15, pp. 3894-3896, 2014.

[39] G. K. P. Lei and M. E. Marhic, "Performance investigation of a hybrid fiber optical parametric amplifier," Optics Express, vol. 21, no. 19, pp. 21932-21940, 2013.

[40] B. P.-P. Kuo and S. Radic, "Highly nonlinear fiber with dispersive characteristic invariant to fabrication fluctuations," Optics Express, vol. 20, no. 7, pp. 7716-7725, 2012. 


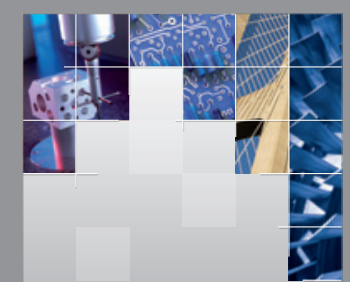

\section{Enfincering}
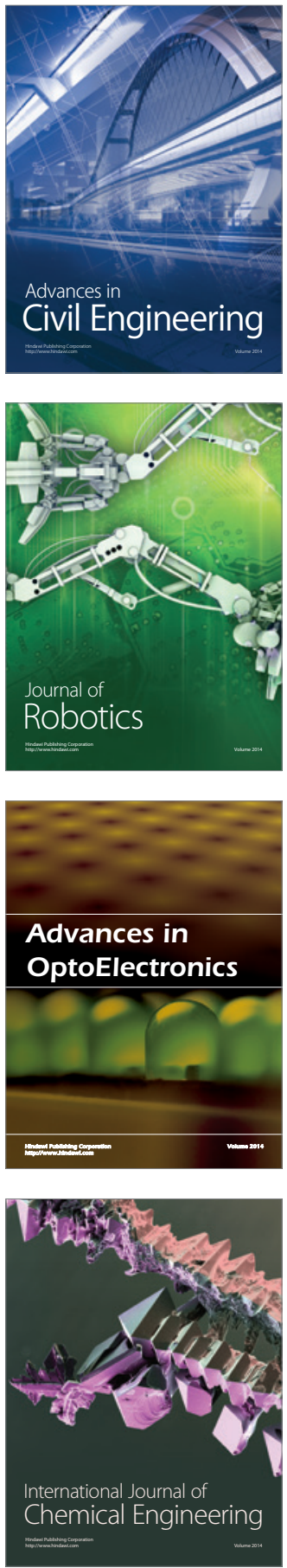

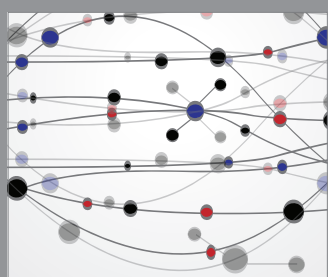

The Scientific World Journal

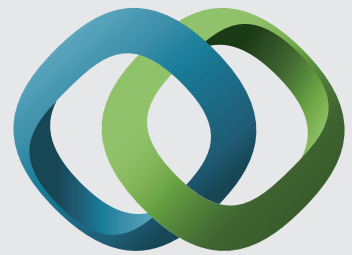

\section{Hindawi}

Submit your manuscripts at

http://www.hindawi.com
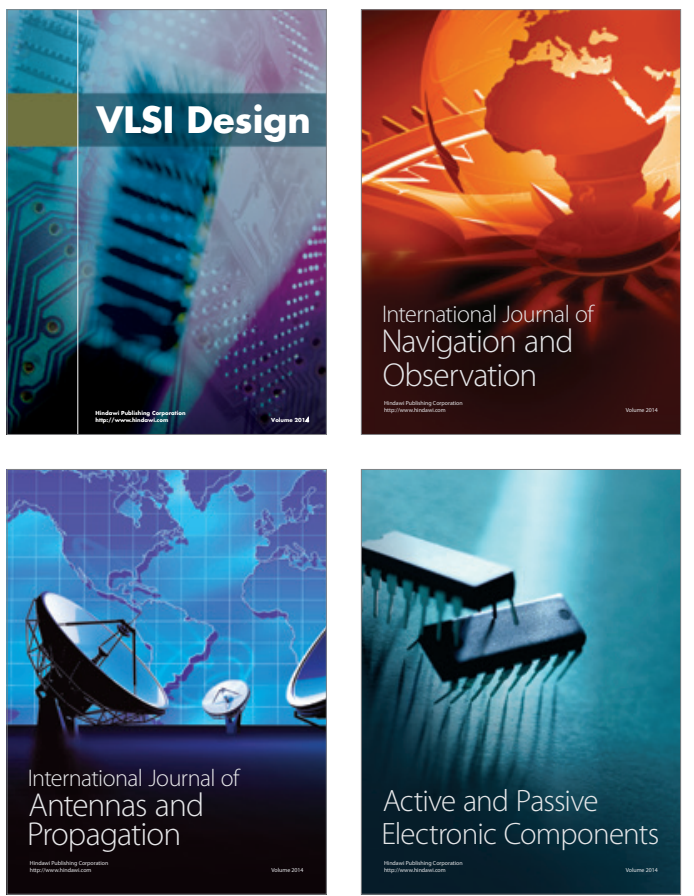
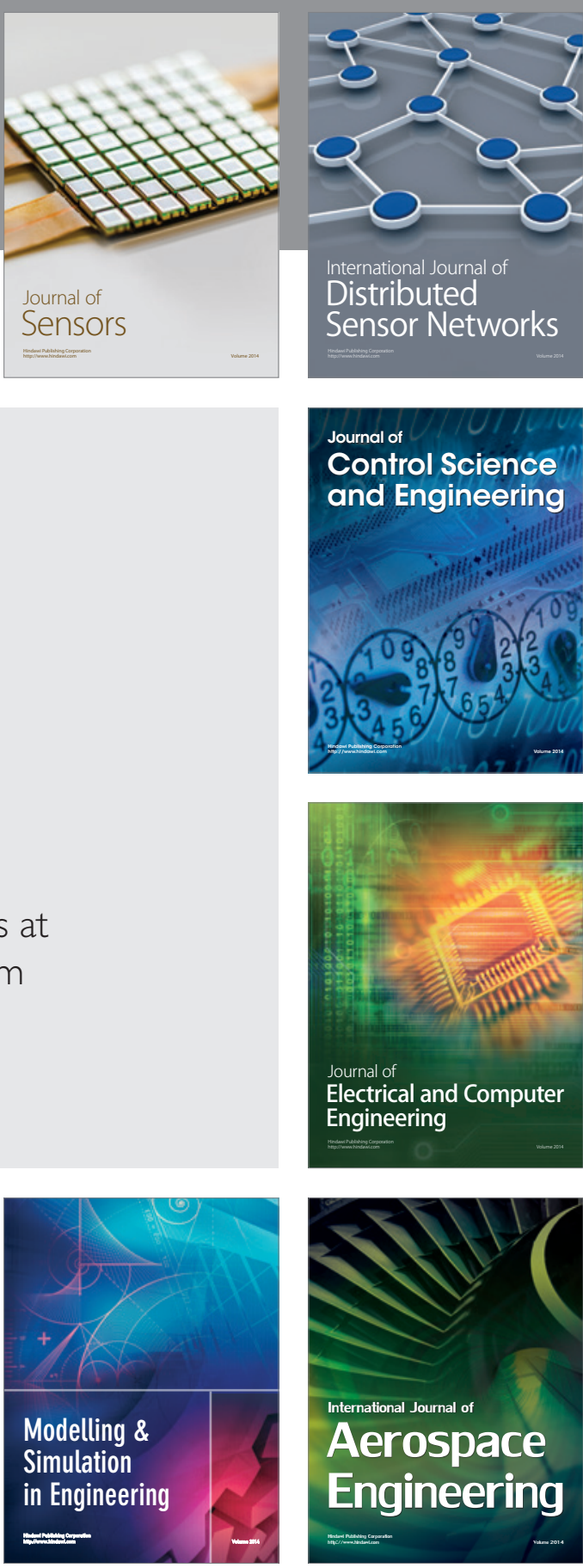

International Journal of

Distributed

Sensor Networks

Journal of

Control Science

and Engineering
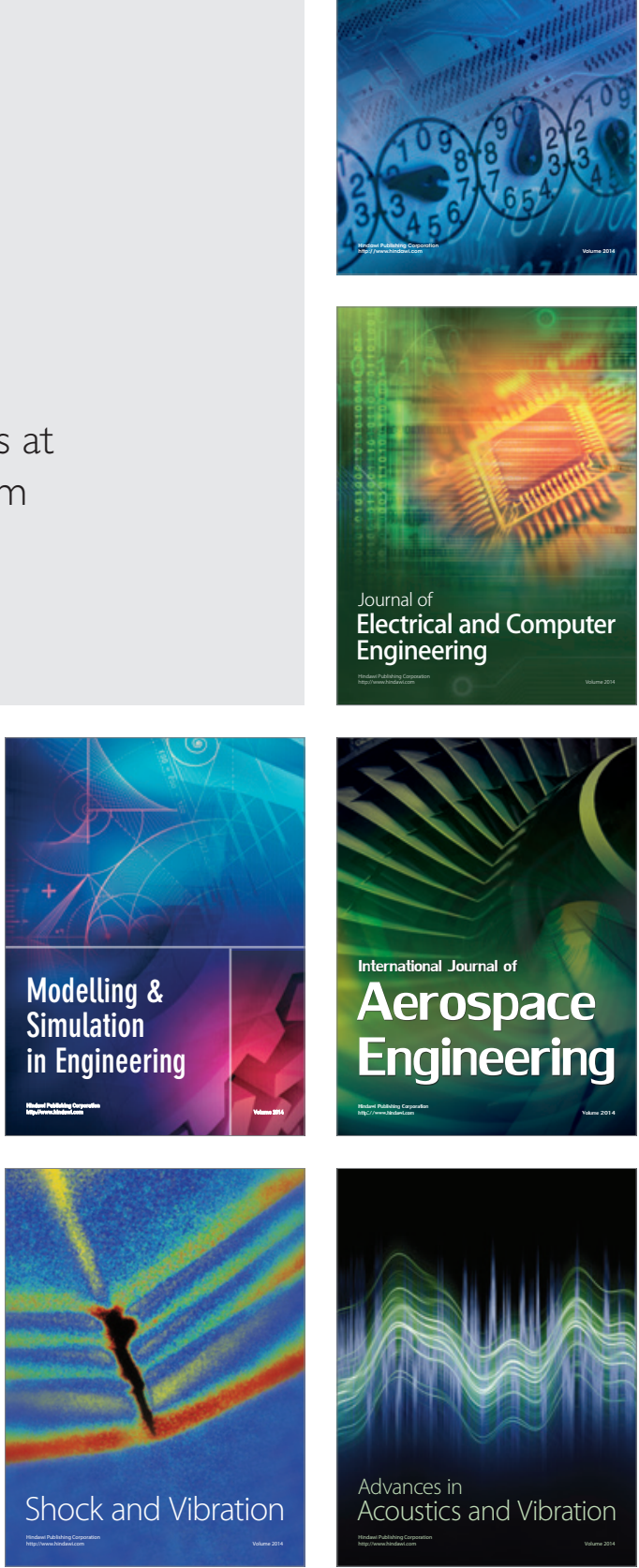